\title{
Foramen tympanicum prevalence in the population of Southeast Brazil: a morphological study in computed tomography scans
}

\author{
T.M.C. Ribeiro1 , A.R. Freire1', E. Daruge Junior², P.R. Botacin³ , F.B. Prado1, A.C. Rossi1 \\ ${ }^{1}$ Department of Biosciences, Anatomy Division, Piracicaba Dental School, University of Campinas, Piracicaba, \\ São Paulo, Brazil \\ 2Department of Social Dentistry, Forensic Dentistry Division, Piracicaba Dental School, University of Campinas, \\ Piracicaba, São Paulo, Brazil \\ ${ }^{3}$ Basic Sciences Department, Araçatuba Dental School, Paulista State University - UNESP, Araçatuba, São Paulo, Brazil
}

[Received: 11 May 2021; Accepted: 20 August 2021; Early publication date: 10 September 2021]

Background: The foramen tympanicum is located on the anteroinferior region of the external acoustic meatus and posteromedial to the temporomandibular joint in children between the $1^{\text {st }}$ and the $5^{\text {th }}$ year of life. It is considered an anatomical variation when it persists in adults. The aim of this study was to verify the prevalence as well as to characterise the foramen tympanicum in computed tomography (CT) scans of the population from southeastern part of Brazil.

Materials and methods: A total of 78 CT scans of dry human skulls (20 female and 58 male) were used, which were selected randomly regarding the ages, ranged from 15 to 100 years. The foramen tympanicum was identified in the images of the axial plane and confirmed in the images of the coronal and sagittal planes. The largest diameter (in $\mathrm{mm}$ ) was obtained. The descriptive statistics (in \%), Fisher's test and $\chi^{2}$ test were performed to compare the prevalence of foramen tympanicum between sexes and sides. The probability value $\leq 0.05$ was defined as a level of significance. Descriptive statistics were performed to verify the mean diameter of the foramen on the right and left sides of the skulls.

Results: The prevalence of foramen tympanicum was higher in females $(p=$ $=0.0070)$, bilaterally, as the absolute values of females were lower in relation to males. Fisher's exact test showed that the prevalence of foramen tympanicum was significantly higher in females (45\%) than in males (15.52\%). On the right side, the mean axial diameter was $2.23 \mathrm{~mm}$ (range $0.93-3.75 \mathrm{~mm}$ ). On the left side, the mean axial diameter was $2.22 \mathrm{~mm}$ (range $0.9-3.61 \mathrm{~mm}$ ).

Conclusions: The knowledge of anatomical variations is extremely valuable for an accurate diagnosis, treatment plan and prognosis and a thorough preoperative assessment. (Folia Morphol 2022; 81, 4: 1042-1046)

Key words: temporomandibular joint, computed tomography, anatomy, foramen tympanicum, Huschke's foramen, tridimensional morphometry

Address for correspondence: A.C. Rossi, PhD, Professor, Department of Biosciences, Anatomy Division, Piracicaba Dental School, University of Campinas, Piracicaba, São Paulo, Brazil, Av. Limeira, 901-Areião, Piracicaba SP, Brazil 13.414-903, tel: 551921065721 ,

e-mail: rossianac01@gmail.com

This article is available in open access under Creative Common Attribution-Non-Commercial-No Derivatives 4.0 International (CC BY-NC-ND 4.0) license, allowing to download articles and share them with others as long as they credit the authors and the publisher, but without permission to change them in any way or use them commercially. 


\section{INTRODUCTION}

The foramen tympanicum (FT), also known as foramen of Huschke, is the bone communication between the external acoustic meatus and the temporomandibular joint (TMJ) found during the development of the tympanic part of the temporal $[1,14]$. The FT is located on the anteroinferior region of the external acoustic meatus and posteromedial to the TMJ in children between the $1^{\text {st }}$ and the $5^{\text {th }}$ year of life. This is considered an anatomical variation when it persists in adults [8]. Topsakal et al. [14] defined the FT as being a variable deficiency in the floor of the bony part.

The formation of FT occurs after birth, when the tympanic ring becomes the tympanic bone and has the shape of a broken ring at its upper extremity. During its development, which occurs in a circular and superior direction through its ossification centres, the tympanic bone will merge with the petrous portion of the temporal bone forming the external wall of the tympanic cavity [12]. The next stage of development is the appearance of two tubers or prominences that grow against each other, until they come together in the first year of life. The tympanic bone presents, because of the fusion of these two tubercles, an opening called the FT [13].

In adults, the presence of FT is associated with the spread of infections originating in the external acoustic meatus, which affect surrounding regions such as the TMJ and the parotid gland; tumours originating in the mandibular fossa of the temporal bone, which uncommonly reach the external acoustic meatus; cases of spontaneous salivary fistula in the external acoustic meatus; and complications in TMJ arthroscopy [8-10]. Finally, the study of FT morphology and prevalence in dry skulls and computed tomography (CT) scans indicated a difference between the sexes [13].

The aim of this study was to verify the prevalence as well as to characterise the foramen tympanicum in CT scans of the population from southeastern Brazil.

\section{MATERIALS AND METHODS}

The research was analysed and approved by the Committee of Research Ethics of the University of Campinas (protocol number CEP-FOP-UNICAMP-CAAE 38866220.3.0000.5418).

\section{Sample}

A total of $78 \mathrm{CT}$ images of human skull selected at random, ranging in age from 15 to 100 years,
20 female and 58 male, and acquired by the Aisteion Multislice 4 CT System (Toshiba Medical Systems Corporation, Japan), for the protocol of the skull: $100 \mathrm{MA}, 120 \mathrm{KV}$, with cuts of $1 \mathrm{~mm}$.

The CT scans were obtained from skulls belonging to the Bone Collection housed at Piracicaba Dental School from the University of Campinas (UNICAMP). The skeletons belong to a contemporary Southeast Brazilian population, are of the actual population and surroundings, are in a good state of preservation, and were identified by death certificates.

The research considered CTs of the skulls that presented the anatomical structures of the temporal bone preserved and intact, without macroscopic deformities. CT scans of individuals with any signs of bone remodelling in the temporal region were excluded, as well as individuals with implants, plates and screws or any other metallic artefact in the referred region.

\section{Foramen tympanicum evaluation using 3D reconstruction}

The Mimics software v. 18.0 (Materialise, NV, Belgium) was used to produce the segmentation of the images on each CT scan. The FT was identified in the images of the axial plane and confirmed in the images of the coronal and sagittal planes. Then, in this same software, three-dimensional (3D) reconstruction was performed to visualise and to confirm the presence of the FT (Fig. 1).

After locating the structure (Fig. 1), the Mimics software v. 18.0 (Materialise, NV, Belgium) was used to measure the largest diameter (in mm). Each FT was evaluated by a single evaluator previously calibrated. The evaluator was trained to determine the presence of the FT, which was evaluated according to its anatomical characteristics observed on CT scans. After training, the measurement was performed using the distance measurement tool, marking the outermost voxel of both ends of the FT, in the axial plane of the computed tomography (Fig. 2).

\section{Statistical analysis}

The data were tabulated in the Office Excel (C) Microsoft 2021). The descriptive statistics (in \%), Fisher's test and $\chi^{2}$ test were performed to compare the prevalence of FT between sexes and sides. The probability value $\leq 0.05$ was defined as a level of significance. Descriptive statistics were performed to verify the mean diameter of the FT on the right 


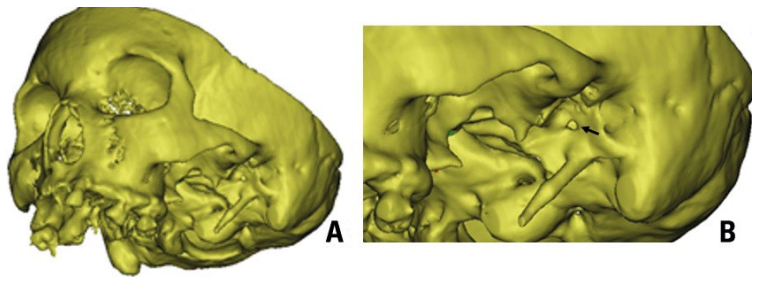

Figure 1. A. Three-dimensional reconstruction of the computed tomography scan of the skull in the Mimics software v. 18.0 (Materialise, NV, Belgium) in a lateroanterior view; B. The black arrow indicates the presence of the foramen tympanicum in a lateral view of the skull.

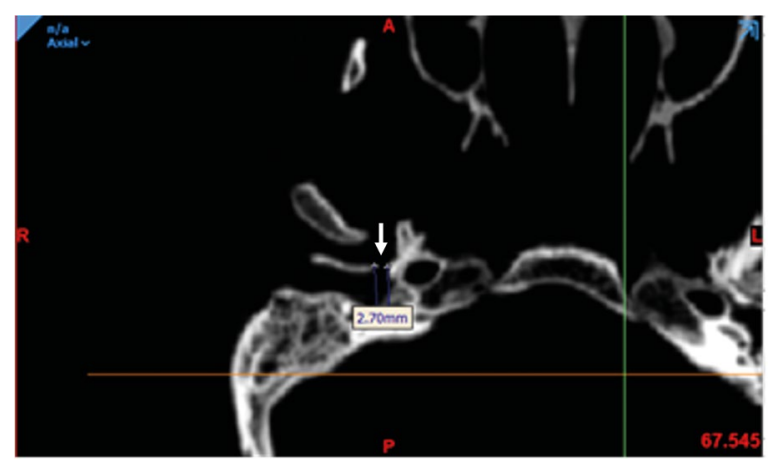

Figure 2. Computed tomography image of the axial plane of the skull, presence of foramen tympanicum indicated by the white arrow. The measurement was obtained using the Mimics software v. 18.0 (Materialise, NV, Belgium).

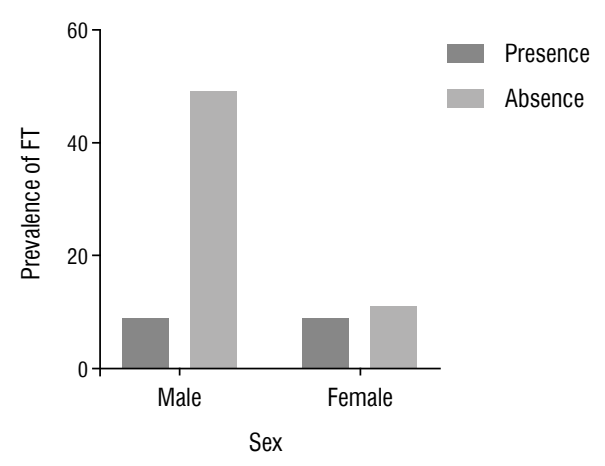

Figure 3. Prevalence of foramen tympanicum (FT) according to the sex.

and left sides of the skulls. All data were analysed using the GraphPAD Prism v.8 software (San Diego, CA, USA).

\section{RESULTS}

In this study, 58 (74.36\%) male and 20 (25.64\%) female skulls were observed. FT was determined in $18(23.1 \%)$ of 78 skulls (they had FT on at least one side of the head). The sample's mean age was
55 years (range: 19-100 years) for females and 54.41 years (range: $15-87$ years) for males.

The $\chi^{2}$ test showed a relationship between sex and FT $(p<0.05)$. The $\chi^{2}$ test showed that the prevalence of FT was higher in females $(p=0.0070)$, bilaterally, as the absolute values of females were lower in relation to males. Fisher's exact test showed that the prevalence of FT was significantly higher in females (45\%) than in males (15.52\%) (Fig. 3).

The FT was bilateral in $14(17.95 \%)$ skulls, 7 in males and 7 in females. On the right side, the mean axial diameter was $2.23 \mathrm{~mm}$ (range: $0.93-3.75 \mathrm{~mm}$ ). On the left side, the mean axial diameter was $2.22 \mathrm{~mm}$ (range: 0.9-3.61 mm). Regarding the unilateral evaluation, we found skulls presenting the FT only on the left side ( 2 female and 2 male skulls).

No correlation between location or sex and diameter of FT was found by unilateral analysis of variance $(p>0.05)$.

\section{DISCUSSION}

Studies in CTs scans indicated this is an extremely important exam for visualising anatomical structures, such as FT [3, 13]. According to Çetin et al. [2], radiological studies, mainly using CT scans, bring contributions to demographic data and dimensions of an anatomical bony feature from a determined population. In a recent study, Topsakal et al. [14] highlighted the importance of visualisation and of the anatomical knowledge of certain anatomical structures, such as the FT, to perform the endoscopic ear surgery. They related that the minimal invasive technological developments such as robot-assisted or image-guided ear surgery claim to be less invasive or more anatomical structure preserving.

Hashimoto et al. [6] believe that there is a difference in prevalence of FT between males and females. From these results, we were interested in investigating if the visualisation of FT in CT scans of dry skulls would be feasible and its prevalence in a sample of Brazilian nationality.

In agreement with Akbulut et al. [1], the present study showed the prevalence of FT of $23.1 \%$. In other studies, the prevalence was approximately $12 \%$ $[3,6,13]$. In a recent study, Deniz et al. [3] examined 200 cone-beam CT images (400 ears) in a Turkish sub-population. The authors noted size and location (unilateral and bilateral) of the present FT and found that the prevalence of the FT was significantly higher in females ( $8 \%$ ) than in males (3.5\%) as well as in the 
present study in a Brazilian sub-population that the persistent FT was significantly higher in females $(45 \%)$ than in males (15.52\%). The literature explained that the female predominance of FT might have been based on growth and development differences of temporal bone between sexes [5].

In relation to the results about side prevalence, Deniz et al. [2] determined FT on the right side in 4\% of patients and FT on the left side in $7.5 \%$ of patients $(p<0.05)$. This was bilateral in $2.5 \%$ of patients. In the present study, FT was bilateral in 14 (17.95\%) skulls, 7 in males and 7 in females. For the unilateral, the results showed the FT on the left side, unilaterally, in 2 female skulls and 2 male skulls. Deniz et al. [2] explained that the relationship between the side and FT can be responsible for mastication habits and genetic factors of study populations. And it is possible to suggest the existence of the corporeal principle of laterality, with one side being more prevalent than the other.

The clinical implications related to the persistence of FT are widely discussed in the literature and the most current are otitis, infectious arthritis, herniation of the disc and fistulas in the external acoustic meatus. TMJ arthroscopy is an exam for diagnosing pathologies in the region, which is performed by a rigid optical fibre with a diameter varying between 1.7 and $2.7 \mathrm{~mm}[5,6,9]$. These studies also showed that severe ear complications can develop during TMJ arthroscopy, due to the lack of preoperative detection of the presence of FT. This could happen because of the similarity between the mean diameter commonly found in FT and the diameter of the optical fibre used during the examination.

Our findings showed that the mean axial diameter for the right side is $2.23 \mathrm{~mm}$ and for the left side is $2.22 \mathrm{~mm}$, corroborating with the literature. In addition, Ertugrul and Keskin [4] concluded that the dimensions of the FT were generally larger in females than in males.

Although CT is an expensive method, high-resolution imaging tests have proved to be a better technique for identifying the presence of FT when compared to conventional radiographs [9]. For instance, in a population with a prevalence of FT of $14.9 \%$, the prevalence was higher in studies on cadavers $(21.2 \%)$ than on radiographs (8.8\%) [2]. Hence, the use of software with the capacity for 3D reconstruction, improved the visualisation of the structure of interest in our study (i.e., the FT). On the oth- er hand, it is important to consider that FT with a diameter of less than $1 \mathrm{~mm}$ may not be visualised in a computed tomography with a slice of $1 \mathrm{~mm}$ thickness [11].

\section{CONCLUSIONS}

In conclusion, the prevalence of FT in CT scans of dry skulls in a Brazilian population sample was $23.1 \%$. Additionally, persistent FT was significantly higher in female (45.0\%) than in male (15.5\%) sex. Further, this study showed that the mean axial diameter for both sides were approximately $2.2 \mathrm{~mm}$. The knowledge of anatomical variations is extremely valuable for an accurate diagnosis, treatment plan and prognosis and a thorough preoperative assessment.

\section{Acknowledgements}

The authors sincerely thank those who donated their bodies to science so that anatomical research could be performed. Results from such research can potentially increase mankind's overall knowledge that can then improve patient care. Therefore, these donors and their families deserve our highest gratitude [7].

\section{Conflict of interest: None declared}

\section{REFERENCES}

1. Akbulut N, Kursun S, Aksoy S, et al. Evaluation of foramen tympanicum using cone-beam computed tomography in orthodontic malocclusions. J Craniofac Surg. 2014; 25(2): e105-e109, doi: 10.1097/SCS.0000000000000440, indexed in Pubmed: 24469373.

2. Çetin H, Akkaşoğlu S, Çalışkan S. New approach to morphometric analysis of Huschkess foramen. Folia Morphol. 2022; 81(3): 749-755, doi: 10.5603/FM.a2021.0056, indexed in Pubmed: 34060643.

3. Deniz Y, Geduk G, Zengin AZ. Examination of foramen tympanicum: an anatomical study using cone-beam computed tomography. Folia Morphol. 2018; 77(2): 335-339, doi: 10.5603/FM.a2017.0078, indexed in Pubmed: 28868610.

4. Ertugrul S, Keskin NK. Relationship of age to foramen of Huschke and investigation of the development of spontaneous temporomandibular joint herniation. Int J Oral Maxillofac Surg. 2019; 48(4): 534-539, doi: 10.1016/j. ijom.2018.08.011, indexed in Pubmed: 30205912.

5. Gonzalez PN, Bernal V, Perez SI. Analysis of sexual dimorphism of craniofacial traits using geometric morphometric techniques. Int J Osteoarchaeol. 2011; 21(1): 82-91, doi: 10.1002/oa.1109.

6. Hashimoto $\mathrm{T}$, Ojiri $\mathrm{H}$, Kawai $\mathrm{Y}$. The foramen of Huschke: age and gender specific features after childhood. Int J Oral Maxillofac Surg. 2011; 40(7): 743-746, doi: 10.1016/j. ijom.2011.03.017, indexed in Pubmed: 21549561.

7. Iwanaga J, Singh V, Ohtsuka A, et al. Acknowledging the use of human cadaveric tissues in research papers: 
Recommendations from anatomical journal editors. Clin Anat. 2021; 34(1): 2-4, doi: 10.1002/ca.23671, indexed in Pubmed: 32808702.

8. Lacout A, Marsot-Dupuch K, Smoker WRK, et al. Foramen tympanicum, or foramen of Huschke: pathologic cases and anatomic CT study. Am J Neuroradiol. 2005; 26(6): 1317-1323, indexed in Pubmed: 15956489.

9. Melgaço C, Penna L, Seraidarian P. O forame de Huschke e suas implicações clínicas. Rev Brasil Otorrinol. 2003; 69(3): 406-413, doi: 10.1590/s0034-72992003000300016.

10. Moreno RC, Chilvarquer I, Hayek JE, et al. Anatomic and radiograph study of the persistence of Foramen of Huschke. Braz J Otorhinolaryngol. 2005; 71(5): 676-679, doi: 10.1016/s1808-8694(15)31273-8, indexed in Pubmed: 16612531.

11. Park YHo, Kim HJ, Park MH. Temporomandibular joint herniation into the external auditory canal. Laryngoscope.
2010; 120(11): 2284-2288, doi: 10.1002/lary.21115, indexed in Pubmed: 20939076.

12. Pękala JR, Pękala PA, Satapathy B, et al. Incidence of foramen tympanicum (of huschke): comparing cadaveric and radiologic studies. J Craniofac Surg. 2018; 29(8): 2348-2352, doi: 10.1097/SCS.0000000000004784, indexed in Pubmed: 30277949.

13. Prowse SJ, Kelly G, Agada F. Temporomandibular joint herniation and the foramen of Huschke: an unusual external auditory canal mass. J Laryngol Otol. 2011; 125(12): 1279-1281, doi: 10.1017/S0022215111002295, indexed in Pubmed: 21910955.

14. Topsakal V, Kachlik D, Bahşi I, et al. Relevant temporal bone anatomy for robotic cochlear implantation: An updated terminology combined with anatomical and clinical terms. Transl Res Anat. 2021; 25: 100138, doi: 10.1016/j. tria.2021.100138. 